Referencia para citar este artículo: Cardona-Rodríguez, F. J., Osorio-Tamayo, D. L. \& Moreno-Carmona, N. D. (2017). La referencialidad, redefinida desde el bienestar subjetivo y la socialización. Revista Latinoamericana de Ciencias Sociales, Niñez y Juventud, 15 (2), pp. 1165-1177. DOI:10.11600/1692715x.1522525102016

\title{
La referencialidad, redefinida desde el bienestar subjetivo y la socialización*
}

\author{
FARLEY JoHANNA CARDona-RodRíGUEZ \\ Profesora Institución Educativa José Acevedo y Gómez, Colombia. \\ DORA LILIANA OSORIO-TAMAYO*** \\ Profesora Universidad Católica Luis Amigó, Colombia. \\ Norman Darío Moreno-CARMona ${ }^{* * * *}$ \\ Profesor Funlam, Colombia.
}

\begin{abstract}
Artículo recibido en octubre 25 de 2016; aprobado en enero 26 de 2017 (Eds.)
\end{abstract}
- Resumen (analítico): se presentan resultados de una investigación realizada con el objetivo definir la referencialidad partiendo del proceso de socialización y la construcción del bienestar subjetivo, en la que participaron 11 jóvenes de 20 a 26 años de Medellín. La modalidad de investigación adoptada fue la teoría fundamentada y los datos fueron obtenidos a través de dos grupos de discusión y cuatro entrevistas semiestructuradas. Como parte de los resultados se destacan las tensiones a considerar para la definición de la referencialidad, su constitución a través de las relaciones significativas que establecen los y las jóvenes con personas dentro y fuera de la familia, y la forma como multiplicidad de relaciones influyen positivamente en la consecución de mejores condiciones de bienestar subjetivo. Las conclusiones dejan ver posibilidades de integración del concepto, construido como orientador de intervenciones psicosociales y de políticas públicas sobre familia y juventud.

Palabras clave: socialización, bienestar, familia, joven, identidad (Tesauro de Ciencias sociales de la Unesco).

\section{Referentiality, redefined from subjective wellbeing and socialization}

- Abstract (analytical): this research study was designed in order to define the concept of referentiality based on the processes of socialization and the construction of subjective wellbeing. The study involved the participation of 11 subjects, aged 20 to 26 from the city of Medellin, Colombia. The research approach used was grounded theory and data was obtained through the holding of two discussion groups and four semi-structured interviews. Some aspects of the results are notable, such as the tensions that are important to consider when defining referentiality, the construction

\footnotetext{
* $\quad$ Este artículo de investigación científica y tecnológica, se basa en una investigación auspiciada por los autores, realizada entre el 10 octubre del 2012 y el 5 de diciembre del 2014, con el fin de optar al título de Magister en Intervenciones Psicosociales de la Fundación Universitaria Luis Amigó. Investigación Cualitativa, teoría fundamentada. Área: Ciencias sociales-Temas especiales.

** Psicóloga. Magíster en Intervenciones Psicosociales (Funlam). Profesora de la Institución Educativa José Acevedo y Gómez. Orcid: 0000-0003-1604-0106. Índice H5: 1. Correo electrónico joacoa@gmail.com

*** Psicóloga. Master en Psicología Analítica (Universidad Ramón Llull-Barcelona). Magister en Intervenciones Psicosociales (Funlam). Profesora de la Universidad Católica Luis Amigó. Orcid: 0000-0003-4639-6445. Índice H5: 1. Correo electrónico: celeste34.liliana@hotmail.com

**** Psicólogo. Magíster en Educación: Desarrollo Humano. Doctor en Investigación Psicológica. Docente de la Funlam. Orcid: 0000-0002-8216-2569. Índice H5: 3. Correo electrónico: normanmorenoca@amigo.edu.co
} 
of referentiality through the meaningful relationships that young people establish with people from both within and outside their family circle, and the way that these multiple relationships positively influence the acquisition of better living conditions that improve subjective wellbeing. The authors' conclusions highlight possibilities for integration opportunities, the creation of a guide for psychosocial interventions and contributions to public policy related to family and youth development.

Key words: socialization, well-being (health), family, young adults, identity (Social Science Unesco Thesaurus).

\section{A referencialidade redefinida desde o bem-estar subjetivo e a socialização}

- Resumo (analítico): o artigo apresenta resultados de uma investigação que tem como objetivo definir a referencialidade a partir do processo de socialização e da construção do bem-estar subjetivo. No estudo participaram 11 jovens que estavam na faixa etária de 20 - 26 anos, todos da cidade de Medellín. O método utilizado foi a teoria fundamentada e os dados foram obtidos através de dois grupos de discussão e quatro entrevistas semiestruturadas. Nos resultados destacam as tensões emergentes na definição da referencialidade, a constituição dela nas relações significativas que estabelecem os jovens com outras pessoas dentro e fora da família, e como a multiplicidade de relações influenciam positivamente a consecução de melhores condições de bem-estar subjetivo. As conclusões mostram as possibilidades de integração do conceito que serve como guia na construção de intervenções psicossociais e de políticas públicas para a família e a juventude.

Palavras chave: socialização, bemestar, família, jovem, identidade (Thesaurus de Ciências Sociais da Unesco).

-1. Introducción. -2. La teoría fundamentada en la construcción de una definición. -3. Individualismo y colectivismo, la experiencia del bienestar. -4. Trayectorias de socialización en la historia familiar. $\mathbf{- 5}$. Otras personas y relaciones significativas en la socialización. -6. Referencialidad como cualidad de las relaciones. -Lista de referencias.

\section{Introducción}

Las dinámicas complejas que ofrece la posmodernidad y sus consecuencias en la vida cotidiana, en las relaciones interpersonales, familiares y sociales, y más especialmente, los cambios en el proceso de socialización de las nuevas generaciones, se constituyen en el punto de partida que da lugar al presente estudio. Como lo evidencian Cardona-Rodríguez, Osorio-Tamayo y Moreno-Carmona (2014), sus implicaciones abren un campo de investigación poco explorado, en especial en el ámbito de la crianza y de las relaciones entre adultos y jóvenes. Por esto, la investigación se centró en comprender cómo estas situaciones influyen en los procesos de socialización de jóvenes de 20 a 26 años de Medellín, quienes provienen de contextos heterogéneos, con altos y continuos contrastes entre los tipos de familia, el acceso recursos y oportunidades y los efectos del conflicto armado y del desplazamiento.
En tanto la referencialidad es un concepto poco explorado y la palabra referente ha tenido un uso indistinto en diversos contextos, la novedad de esta investigación estuvo en lograr comprensiones que partieron de la reflexión de los mismos jóvenes sobre su propia historia y ampliaron la discusión sobre el concepto de referentes, desde el proceso de socialización y el bienestar subjetivo.

Se seleccionó la Teoría Fundamentada con la intención de tomar como datos básicos las formas de vida y las concepciones cotidianas de las personas. En esta vía, el artículo presenta la metodología, los hallazgos relevantes en el proceso de codificación y una discusión entre las categorías emergentes y algunos conceptos en tensión. Al finalizar, se hace una aproximación a una teoría sustantiva sobre la referencialidad, se introduce el concepto de red referencial y se enuncian efectos favorables para la socialización de jóvenes relacionadas con este tipo de vinculación. 
En las conclusiones se destaca la importancia de promover, proveer y mantener redes referenciales que aporten a la construcción del bienestar subjetivo, como orientaciones para las intervenciones psicosociales y el desarrollo de políticas públicas.

\section{La teoría fundamentada en la construcción de una definición}

Al tomar las narraciones de los y las jóvenes como el material básico para intentar definiciones de referencialidad, se tuvo en cuenta la concepción de definir, en la dirección de hacer claridad sobre las significaciones y sentidos emergentes en las experiencias, las valoraciones y las reflexiones presentadas por ellos. Sus palabras, asignaciones y verbalizaciones fueron utilizadas como datos básicos para la construcción de conceptos. Este sentido coincide con las definiciones de Teoría Fundamentada, aportadas por Strauss y Corbin (2002) y Galeano (2004), en las que se destaca la importancia de la información procedente de los participantes, con el objetivo de lograr nuevas comprensiones de los fenómenos psicosociales, a través de la generación teorías que como productos se parezcan mucho más a la realidad. El proceso investigativo es definido como un ejercicio sistemático en el que se mantiene una relación estrecha entre "la recolección de datos, el análisis y la teoría que surgirá de ellos" (Strauss \& Corbin, 2002, p. 21).

La indagación de antecedentes evidenció vacíos con relación a la constitución de la referencialidad como una categoría delimitada en ciencias sociales ${ }^{1}$. Los procesos de codificación se orientaron a ganar en densidad y abstracción, para comprender y describir el fenómeno, asociando los conceptos extraídos de las reflexiones y definiciones aportadas por los participantes.

La selección de los informantes se realizó considerando una la población con capacidad de expresar posiciones personales frente a sus trayectorias de vida y la construcción de su

1 Las orientaciones conceptuales de la investigación, se publicaron en el artículo Construcción de la referencialidad en las actuales dinámicas de socialización (Cardona-Rodríguez, OsorioTamayo \& Moreno-Carmona, 2014) bienestar, más allá de las manifestaciones de estados de ánimo momentáneos; de modo que a través de valoraciones retrospectivas y cercanas frente a sus posiciones y relaciones, se facilitara la identificación de referentes en los vínculos familiares y sociales relevantes para ellos. Esta descripción coincide con la realizada por Arias, Arias, Jaramillo, Restrepo y Ruiz (2009), en una investigación sobre socialización con jóvenes universitarios entre 20 a 26 años, por lo cual se seleccionó este rango de edad.

Otro aspecto que orientó las decisiones sobre la muestra, fue el interés de contrastar escenarios de socialización con características, repertorios relacionales y posibles concepciones del bienestar diferentes, que dieran cuenta, en parte, de la heterogeneidad de trayectorias de vida de los y las jóvenes de la ciudad. Las personas fueron seleccionadas por conveniencia y siguiendo los planteamientos del muestreo teórico, en cuanto permite convocar a los participantes en el momento en que se precise de información específica requerida para ampliar los conceptos en desarrollo. En total participaron en la investigación 11 jóvenes, 5 hombres y 6 mujeres,

Consecuente con las características metodológicas descritas, se utilizaron para la recolección de información, grupos de discusión y entrevistas semiestructuradas. Los primeros permitieron contrastar la concepción del bienestar y los procesos de socialización de jóvenes del Centro de Desarrollo Cultural de Moravia $(\mathrm{CDCM})^{2}$ y estudiantes de universidades privadas, en etapa de desarrollo de prácticas profesionales. Adicionalmente los grupos permitieron identificar informantes clave para la etapa de profundización en los conceptos emergentes.

Tras la codificación inicial, se hizo necesaria nueva información, que permitiera amplificar las dimensiones y características de los códigos

El Centro de Desarrollo Cultural es una iniciativa de la Alcaldía de Medellín, que fortalece las capacidades de participación y brinda formación artística, cultural y en emprendimientos a jóvenes del Barrio Moravia. El barrio está en una zona de la ciudad, producto de asentamientos y procesos sociales conflictivos, siendo uno de los más importantes la ubicación y posterior traslado del basurero municipal, entre los años 1977 y 1984. En época reciente ha sido centro de reestructuración urbana, implicando la reubicación de algunas familias en otros sectores de la ciudad (Quiceno, Cardona \& Montoya, 2006). 
que fueron surgiendo. Para recolectarla se realizaron cuatro entrevistas semiestructuradas, en las que se hizo énfasis en las expresiones y explicaciones de los participantes, sobre sus conocimientos y experiencias, con lo cual los códigos ganaron en profundidad y se hicieron más accesibles a la interpretación (Flick, 2004).

La construcción de las categorías teóricas fue el resultado de la comparación entre incidentes y conceptos emergentes, siguiendo las indicaciones propuestas por Strauss y Corbin (2002) en la descripción del método comparativo constante. La comparación de incidentes y códigos generó nuevas asociaciones e incluso recodificaciones, hasta llegar a la conformación de redes de significados que se asociaron en tres mapas conceptuales: socialización, definición de bienestar y cualidades de referente. Este ejercicio mostró las necesidades de tener en cuenta a jóvenes con otros tipos de familia, en procesos de resocialización o rehabilitación e involucrar hombres.

Al evidenciar en los códigos precisión y suficiencia se finalizó la indagación, teniendo en cuenta el concepto de saturación teórica, definido como aquel momento en el que los datos no arrojan nueva información desde la cual se posibilite profundizar o ampliar la construcción de la teoría (Carrero, Soriano \& Trinidad, 2012).

\section{Individualismo y colectivismo: la experiencia del bienestar}

Las percepciones y valoraciones que manifestaron los y las jóvenes, respondieron inicialmente a preguntas sobre las condiciones de vida que relacionaban con estar bien. Las definiciones y experiencias aportadas incluyeron comparaciones entre momentos de su vida, su condición propia frente a la condición de otros sujetos o grupos de jóvenes, $\mathrm{y}$ entre las situaciones de su contexto y otros contextos cercanos.

Cuando se observan las definiciones emergentes, se pueden reconocer dos tendencias. De un lado, valoran las condiciones del contexto como muy influyentes en su bienestar, atribuyendo factores que afectan de manera general y de forma similar a todas las personas de su barrio o grupo social; entre los que se encuentran el acceso a educación, a las oportunidades para el trabajo y a fuentes de ingreso, la suficiencia en los recursos para cubrir las necesidades y el mantenimiento de buenas condiciones de salud. De modo que para el grupo de jóvenes del CDCM, la garantía de acceso a satisfactores y a los medios económicos para el bienestar se convierten en aspiraciones más o menos colectivas, asociando su consecución tanto al mérito personal, como resultado de la construcción conjunta de la comunidad a la que pertenecen: "el bienestar lo construimos entre todos" (Informante 1). Dentro de las aspiraciones compartidas, se esperan mejores condiciones de seguridad $\mathrm{y}$ tranquilidad, siendo los efectos del conflicto urbano una preocupación y una amenaza.

En la otra tendencia, las valoraciones personales son la base para estar bien, partiendo de las actividades y oportunidades para el disfrute, que en sentido más individual que colectivo, resaltan la importancia de los gustos y satisfactores propios: las prácticas deportivas, el acceso a actividades de esparcimiento y diversión, la posibilidad de obtener bienes materiales diversos y la manifestación del deseo de elegir, en sus propias palabras "hacer lo que te guste". Estas posiciones individualistas fueron más evidentes en el grupo de jóvenes universitarios, caracterizados como grupo más por ser estudiantes que por compartir un contexto común. En gran parte, sus familias les han provisto de medios para estudiar y no manifiestan insatisfacción frente a necesidades básicas. Sus ideas del bienestar están orientadas a logros personales, en especial estabilidad emocional y autosuficiencia económica.

Estas condiciones hacen pensar que, contextos de oportunidades diferentes tienen implicaciones particulares frente a la posición desde la cual cada joven asume lo que le hace bien o no, ubicándose en mayor o menor medida, entre el interés por mejorar las condiciones de su comunidad o grupo y las obtención de satisfactores individuales.

La idea del bienestar relacionado con la posibilidad de tomar decisiones por sí mismos, es un aspecto en común entre ambas tendencias; las decisiones se relacionan con qué estudiar, 
en qué trabajar, cómo divertirse, qué valores asumir $\mathrm{y}$, en general, qué hacer con la propia vida.

La relación bienestar y capacidad de elección lleva a los y las jóvenes a referirse a la satisfacción como una experiencia subjetiva, aunque influenciada por el contexto; en este sentido, surge reiteradamente la apreciación sobre la posición y valoración personal como resultado del juicio que cada uno hace de su contexto; en palabras de uno de los jóvenes "todo es como muy psicológico, todo va en la mente, en la mente de la persona...", y contrario a lo expresado anteriormente, pareciera que las motivaciones frente el bienestar no se pueden generalizar.

Ahorabien, frente ala influenciadelcontexto como medio para la búsqueda de bienestar, se construyeron sentidos diversos, desde la realización de actividades de satisfacción personal, evidente en los universitarios, hasta el insertarse en actividades de participación colectiva, que amplían sus vínculos, posición más cercana al grupo de Moravia. Aparece una contraposición en las ideas de bienestar subjetivo, entre los recursos individuales y la relación con el contexto (Vielma \& Alonso, 2010), pero además, entre la tendencia a buscar estar bien desde el apoyo colectivo o desde el esfuerzo y superación personal, tendencias que puede coexistir en los grupos humanos (Bilbao, Techio \& Paéz, 2007), o incluso ser alguna más preponderante en unas culturas $\mathrm{u}$ otras (Carballeira, González \& Marreo, 2015).

En las entrevistas se evidencia como actitudes colectivistas e individualistas parecen contribuir de distinta manera al bienestar. Se presentan diferencias de significado entre quienes reportan acceso a un mayor número de relaciones durante su crecimiento, en familias ampliadas, en las cuales los logros asociados al bienestar son intereses colectivos, $\mathrm{y}$ aquellos que reportaron relaciones limitadas en familias reducidas, donde los padres son figuras centrales, refiriendo la superación de los problemas al esfuerzo individual de la familia y de sus miembros, aquí el aislamiento social es una fuente de malestar; idea que coincide con lo planteado por López-Sánchez (2015).
Entre la superación personal e individual y el soporte social que amplía el circulo a otros con quienes se construye e interactúa de manera más comunitaria, se muestra una de las tensiones que enmarca la definición del concepto (Carballeira et al., 2015). La referencialidad se establece entre las interacciones de reconocimiento en la colectividad y la construcción de salidas restringidas a los esfuerzos del individuo.

En cualquiera de los dos casos, los referentes se identificaron como relaciones que permitieron reconocimiento y apoyo, de los padres o de otras personas que brindaron acciones positivas, desde la familiaridad ampliada. En éstos últimos, además se mantiene un sentido de pertenencia a un grupo más amplio, que provee oportunidades de reconocerse y proyectar sus metas individuales. Tal como mencionan Montoya-Zuluaga, Castaño-Hincapié y Moreno-Carmona (2016), el soporte social y las acciones protectoras de adultos cercanos, pueden constituir recursos alternativos frente a la ausencia o conflicto con los padres.

Las valoraciones sobre qué es estar bien, se van estableciendo por oposición entre tendencias colectivistas y exigencias individualistas, entre las influencias del contexto y la independencia de las valoraciones personales. Consecuente con esto, la referencialidad aparece como una cualidad de aquellas relaciones que posibilitan a los y las jóvenes soportar la contradicción frente a las condiciones del contexto y las concepciones de logro según las expectativas de su comunidad, de su familia y de ellos mismos.

\section{Trayectorias de socialización e historia familiar}

Las trayectorias que se relatan a continuación resultaron de una selección intencionada de casos, presentan estructuras familiares distintas, que ejemplifican algunos tipos del polimorfismo familiar colombiano (Páez-Martínez, 2016). En éstas se destacan, logros y dificultades particulares, valoraciones sobre momentos difíciles y de satisfacción, enmarcando relaciones que les han aportado a sus condiciones de bienestar actual. 
En las entrevistas, la atención se dirigió a las personas significativas que según el joven han aportado a su bienestar. Los cuatro entrevistados relacionaron capacidades y aprendizajes que les han permitido estar bien con la influencia de sus padres, ellos ocupan un lugar importante, tanto desde sus aportes como si han estado ausentes en esta construcción.

Cada historia familiar ofrece diferentes panoramas relacionales, siendo los relatos sintetizados aquí el efecto de las ausencias, las pérdidas y los abandonos a los que estuvieron expuestos, a la vez que destacan acompañamientos, presencias y apoyos recibidos durante su vida, desde ambos valoran sus vivencias en general, resaltando aquellas que han aportado a la construcción de bienestar. Las dos historias condensadas en la Tabla 1, evidencian como la violencia política y social vivida en el país y la ciudad, han alterado las estructuras familiares, modificando los procesos de socialización durante la infancia y la adolescencia.

Tabla 1: Trayectorias inmersas en condiciones violentas.

\begin{tabular}{|c|c|c|c|}
\hline Categorías & Trayectorias & Bienestar & Referentes \\
\hline $\begin{array}{l}\text { Informante } 1 \\
\text { Mujer }\end{array}$ & $\begin{array}{l}\text { El padre fue asesinado cuando } \\
\text { era muy pequeña. La familia } \\
\text { vivió primero en la comuna } 13 \text {, } \\
\text { luego fueron desplazados por } \\
\text { las condiciones de seguridad, } \\
\text { y fueron a Moravia, buscando } \\
\text { mejores condiciones de vida. } \\
\text { La madre se vuelve a casar, } \\
\text { tiene } 2 \text { hijos varones, convive } \\
\text { con ellos y su esposo. }\end{array}$ & $\begin{array}{l}\text { Esta joven, declara que no le } \\
\text { hizo falta tener la figura de un } \\
\text { padre, no manifiesta nostalgia } \\
\text { por su ausencia y no reconoce } \\
\text { en su proceso de socialización } \\
\text { una figura paterna que le } \\
\text { sirviera de referente, ni siquiera } \\
\text { su padrastro }\end{array}$ & $\begin{array}{l}\text { La mayor parte de la infancia } \\
\text { la pasó con una tía, que se } \\
\text { hacía cargo del cuidado de sus } \\
\text { hijos y sus sobrinos, mientras } \\
\text { los otros miembros de la } \\
\text { familia, incluida la madre de } \\
\text { la informante, trabajaban para } \\
\text { sostenerse. }\end{array}$ \\
\hline $\begin{array}{l}\text { Informante } 4 \\
\text { Varón }\end{array}$ & $\begin{array}{l}\text { Huérfano a muy temprana } \\
\text { edad, con pérdidas asociadas } \\
\text { a la violencia, incluyendo el } \\
\text { suicidio de su padre biológico, } \\
\text { que estaba en prisión y el } \\
\text { asesinato de su madre cuando } \\
\text { él tenía dos años. Se trasladaron } \\
\text { de Caucasia a Yarumal y } \\
\text { finalmente en Medellín, } \\
\text { huyendo del conflicto. La } \\
\text { abuela materna se hizo cargo } \\
\text { de él, ella tenía una relación } \\
\text { de pareja muy violenta. A la } \\
\text { agresión al interior de la familia } \\
\text { se le sumó el desplazamiento } \\
\text { forzado, hecho significativo } \\
\text { que marcó la vida familiar. }\end{array}$ & $\begin{array}{l}\text { El compañero de la abuela } \\
\text { consumía marihuana, haciendo } \\
\text { esta droga accesible para el } \\
\text { niño. En su mente infantil } \\
\text { de ocho años, relacionó este } \\
\text { hábito con la alegría de aquel } \\
\text { hombre, por lo cual él mismo } \\
\text { utilizó la droga para hacerse } \\
\text { feliz. Tenía } 8 \text { años, la abuela } \\
\text { se enteró del consumo unos } 9 \\
\text { años después. } \\
\text { Durante la entrevista se } \\
\text { nombra recuperado, tras un } \\
\text { proceso de rehabilitación de } \\
\text { dos años. En este tiempo, ha } \\
\text { llegado a pensar que su vida } \\
\text { sería otra si su madre hubiese } \\
\text { estado con él. Siente que ha } \\
\text { recuperado formas de relación, } \\
\text { más amorosas y solidarias con } \\
\text { su madre-abuela. }\end{array}$ & $\begin{array}{l}\text { Construyó una relación } \\
\text { padre-hijo con el compañero } \\
\text { sentimental de su madre, padre } \\
\text { de su hermano menor; pero } \\
\text { éste también fue asesinado. } \\
\text { Durante este tiempo el joven se } \\
\text { debatió entre el reconocimiento } \\
\text { de la abuela como de madre, y } \\
\text { la nostalgia de haber perdido } \\
\text { su propia madre. }\end{array}$ \\
\hline
\end{tabular}

Fuente: elaboración propia.

Las trayectorias consignadas en la Tabla 2, revelan influencias opuestas en la constitución familiar. En la primera confluyen la ilusión de ser la familia ideal con las relaciones violentas entre los padres, aquí los roles paternos son definidos de manera fija y se asumen como 
únicos responsables del cumplimiento de la felicidad. En la segunda, un hijo que nace tras una relación casual, alrededor de quién se constituye la familia posteriormente, con la inclusión de abuelos y tíos paternos encargados de su cuidado.

Tabla 2: Trayectorias en familia nuclear y extensa.

\begin{tabular}{|c|c|c|c|}
\hline Categorías & Trayectorias & Bienestar & Referentes \\
\hline $\begin{array}{l}\text { Informante } 2 \\
\text { Mujer }\end{array}$ & $\begin{array}{l}\text { Hija única, sus padres } \\
\text { cohabitan, sólo por el amor } \\
\text { hacia su hija. La violencia } \\
\text { asociada al consumo de } \\
\text { alcohol del padre, marcó la } \\
\text { mayor parte de su infancia, De } \\
\text { niña observaba constantemente } \\
\text { las agresiones, no recuerda que } \\
\text { recibieran soporte o ayuda de } \\
\text { otras personas para resolver su } \\
\text { situación. }\end{array}$ & $\begin{array}{l}\text { Tras algunas confrontaciones } \\
\text { entre los padres, la madre } \\
\text { le preguntaba por sus } \\
\text { sentimientos y le prometía que } \\
\text { "todo iba a estar bien". } \\
\text { Una forma infantil de asumirlo } \\
\text { era repetirse para sí misma que } \\
\text { todo eso pasaría, imaginarse } \\
\text { que se encontraba en otros } \\
\text { lugares y recordar que lo más } \\
\text { importante era que su familia } \\
\text { permaneciera unida. } \\
\text { Actualmente tiene mejores } \\
\text { relaciones con su padre que } \\
\text { con su madre, y una mayor } \\
\text { claridad del aporte que éste } \\
\text { hizo a su bienestar. }\end{array}$ & $\begin{array}{l}\text { Aparte de los padres, la } \\
\text { abuela materna es la figura } \\
\text { más significativa, aunque } \\
\text { no la relaciona con acciones } \\
\text { protectores, niatenuantes de los } \\
\text { efectos de la violencia entre sus } \\
\text { padres sobre ella; es posible que } \\
\text { la abuela ni siquiera estuviera } \\
\text { muy informada. Sin embargo, } \\
\text { recuerda como significativo, } \\
\text { que pasaba mucho tiempo con } \\
\text { la ella, describe su relación } \\
\text { como empática y amorosa. } \\
\text { A pesar de la presencia de } \\
\text { otras cuidadoras, debido al } \\
\text { trabajo de ambos padres, éstas } \\
\text { no representaron vínculos } \\
\text { significativos. }\end{array}$ \\
\hline $\begin{array}{l}\text { Informante } 3 \\
\text { Varón }\end{array}$ & $\begin{array}{l}\text { Primogénito de sus padres, } \\
\text { quienes se involucraron en } \\
\text { una relación ocasional. Sus } \\
\text { primeros cuatro años los vivió } \\
\text { con la mamá en otra ciudad. } \\
\text { Luego de algunas situaciones } \\
\text { confusas, el padre se trae al } \\
\text { hijo a vivir a Medellín; durante } \\
\text { un tiempo la familia paterna } \\
\text { impidió cualquier contacto } \\
\text { con la madre. A su padre lo } \\
\text { veía como alguien bastante } \\
\text { distante, como una autoridad; } \\
\text { sólo el último año han logrado } \\
\text { un acercamiento afectivo. Las } \\
\text { dificultades con la esposa del } \\
\text { padre, hacen que vuelva con } \\
\text { su abuela, pero poco tiempo } \\
\text { después ella emigra a otro país; } \\
\text { esta situación hace que quede a } \\
\text { cargo del abuelo, un tío y una } \\
\text { tía. }\end{array}$ & $\begin{array}{l}\text { El reencuentro con la madre fue } \\
\text { en la pre-adolescencia, lo unen } \\
\text { a ella sentimientos de amor y } \\
\text { reconocimiento, entiende su } \\
\text { vida como muy dura y tiene } \\
\text { una idea del sufrimiento que } \\
\text { tuvo al separase de él. } \\
\text { Frente a su familia expresa: } \\
\text { "mi familia... ahora que me } \\
\text { lo preguntas, yo sabía que en } \\
\text { sí no es que no tuviera familia, } \\
\text { si no que todos estábamos } \\
\text { separados". }\end{array}$ & $\begin{array}{l}\text { Una situación de abuso sexual, } \\
\text { vivida en la primera época al } \\
\text { llegar a Medellín, a los } 5 \text { años, } \\
\text { aparece como una influencia } \\
\text { importante en su orientación } \\
\text { sexual. } \\
\text { Es muy significativa su } \\
\text { abuela paterna. Luego, en el } \\
\text { tiempo que vivió con el padre, } \\
\text { resalta las dificultades de la } \\
\text { convivencia con su madrastra, } \\
\text { a pesar de las cuales le } \\
\text { reconoce algunas enseñanzas } \\
\text { importantes para su vida. } \\
\text { Entre los demás cuidadores, su } \\
\text { relación más significativa fue } \\
\text { con el tío, que también migró } \\
\text { un tiempo después. }\end{array}$ \\
\hline
\end{tabular}

Fuente: elaboración propia.

En estas trayectorias aparece una ampliación de las relaciones de soporte, sobre personas distintas a los padres, se comparte la nostalgia por una estructura constituida por un padre, una madre y, en uno de los casos, por los hermanos; en sus relatos la formación brindada 
por estas personas tendría que favorecer aún más la constitución del bienestar; en este sentido las ausencias de las figuras paternas se ven bajo el lente de algo que debió ser de otra manera. Este aspecto emergente del deber ser de la familia, permitió reconocer el peso que tiene sobre su experiencia la ilusión de familia:

“... claro, siempre debe de haber una figura paterna... en una familia tienen que estar los dos, tanto la figura materna como paterna" (Informante 2).

"... yo creo que es como en la etapa del desarrollo que es fundamental tener esas figuras, por lo menos esa figura materna pero, de ahi en adelante, fundamental no es" (Informante 1).

En estas expresiones se nota la dificultad de desvincular las creencias sobre las formas ideales de familia de las expectativas que tienen frente a su propio bienestar; no se puede negar que los padres adquieren un valor fundamental, la dificultad estriba en lo abstracto de las respuestas frente a de qué manera o cómo contribuyeron; lo común es atribuir la presencia o ausencia de los padres el bienestar o el malestar, enfatizando en su deber de haber estado o de comportarse de otra manera.

Se evidencia a los padres como receptores de la proyección de un comportamiento apto, como anhelo de haber recibido otro acompañamiento o la nostalgia por su ausencia, relacionada con la idea que ellos hubiesen sido mejores cuidadores. La aceptación de los padres con sus errores es una perspectiva que, en algunos casos, ha implicado reconocerles en su simple humanidad.

Comprender la familia, más allá de la disfuncionalidad o de la idea de que algo anduvo mal en aquellas que no se correspondieron con un ideal biparental, patriarcal o nuclear, es un planteamiento en algunas teorías psicológicas y sociales (Hillman, 1998; Valdivia, 2008). Sin embargo, la responsabilidad que tienen los padres sobre el bienestar e incluso la salud mental de sus hijos, sigue estando presente en los discursos tanto de jóvenes, como de profesionales y educadores. Si bien se podría pensar que este sentimiento es individual y resulta de la falta real o el distanciamiento emocional, en los relatos se evidenció que estas concepciones aparecen como ideas dominantes, respaldadas por creencias colectivas $y$ reforzadas por los grupos de pertenencia.

\section{Otras personas y situaciones significativas en la socialización}

Al asumir la vida comunitaria, reconocerse en grupos de referencia y hacer un tránsito institucional, las trayectorias varían rápidamente; la vida de los y las jóvenes enfrenta otras situaciones que como el conflicto armado, el desplazamiento, y los efectos de la hibridación cultural, hacen parte de la vida de la Medellín contemporánea; generándose relaciones en las que, tal como lo plantean algunos autores, otros referentes aparecen de manera temprana e incluso intempestiva frente al contexto familiar (Gergen, 2010; ErazoCaicedo \& Duitama-Zamora, 2015).

El conflicto de las dos últimas décadas en la ciudad de Medellín ha tenido efectos heterogéneos y con diferentes impactos en las trayectorias de los participantes. En dos casos aparece la violencia urbana y el desplazamiento forzado, como fuentes de malestar y límite para la construcción de mejores condiciones de vida.

En su experiencia, una joven destaca el esfuerzo que implicaba continuar con los ritmos de vida, complicados por la presencia de actores armados en la Comuna 13 de Medellín y la consecuente tensión e inseguridad (informante 1). Las pérdidas en contextos de violencia afectan las posibilidades de relación, un joven reporta que gran número de sus amigos fueron asesinados (informante 4).

Una de las inquietudes que surge a partir de estas historias es de qué manera han pasado estos jóvenes de unas realidades objetivas de tanta dificultad a la valoración subjetiva de estar bien. En ninguno de los casos se pueden negar las afectaciones del conflicto, pero ambas entrevistas también dan cuenta de valoraciones positivas.

"Yo creo que por el hecho de haber pasado por esa situación tan maluca en la Comuna 13, eso en cierto modo como que ayuda a uno a movilizarse, a tomar acciones frente a eso que está pasando y no simplemente de echarse a morir y a 
llorar porque ya no tiene un montón de seres al lado" (Informante 1).

"Más bien desde ahi empecé a pensar, vamos a vivir más, vamos a hacer más cositas, vamos a hacer cosas buenas; por ejemplo en el momento yo trabajo, estudio y después de tanto tiempo es la primera vez que no tengo enemigos encima" (Informante 4).

En estas experiencias, la referencialidad surge en la tensión entre la realidad del contexto y la valoración subjetiva de los y las jóvenes. Los tipos de moratoria dan cuenta del contraste; mientras el grupo de Moravia resalta el trabajo y la subsistencia como fines del bienestar, el grupo de universitarios se enfoca en la obtención de bienes y logros personales. Las diferencias también delatan la heterogeneidad de la ciudad, de sus espacios de socialización, de la disposición desigual de oportunidades y de la compleja estructura de representaciones y relaciones colectivas, que proveen ideales diversos de estar bien.

De hecho, si se tiene en cuenta las características de la vida juvenil de Medellín, (Alcaldía de Medellín, 2003), que se destaca por un $50 \%$ de población habitante en zonas populares, caracterizadas por condiciones socioeconómicas bajas, con menor acceso a la educación superior, alta deserción escolar y vinculación temprana al mercado laboral, se hace difícil pensar cómo el bienestar pueda estar más asociado a valoraciones personales que a condiciones objetivas.

La idea del bienestar como un aspecto subjetivo, aparece en las narraciones de los y las jóvenes en tanto cada uno podría definir que está bien o no, dependiendo de lo que espera o quiere de su vida. Altas expectativas en contextos de pocas oportunidades, hacen surgir más fácilmente la idea de que las cosas no están bien, tal como afirman Gómez, Villegas, Barrera y Cruz (2007). Sin embargo también se ha anotado que en estos contextos el bienestar aumenta si han una proyección de metas más allá de sí mismos (Carballeira et al., 2015).

Podría decirse entonces que la ampliación de las relaciones incide en las oportunidades para el bienestar, adquiriendo importancia los lazos familiares extendidos a otras personas y cuidadores diferentes a los padres, así como las relaciones establecidas en la escuela y los grupos sociales, que favorecen realizar diversas actividades de interés. Los siguientes testimonios muestran vínculos relacionales amplios que sirvieron de soporte para la construcción de bienestar, algunas personas estaban ahí, ofrecían cuidados y se constituyeron en relaciones significativas:

“... Y mi abuela siempre me daba el puesto del niño de la casa, porque yo no soy hijo de ella, pero soy el hijo del primer hijo de ella, y soy como lo último, pues de hecho, yo soy el primer nieto y el mayor, y aun asi grande, marcado se ve como es ella conmigo y como es ella con los otros nietos... eso que me hizo sentir pues como querido, [en el lugar] que mi papá nunca me dio" (Informante $3)$.

"Lo que pasa es que yo viví, o sea, toda la vida viví con mi tía prácticamente, hasta que nos fuimos de San Javier, porque mi mamá todo el tiempo se la pasaba trabajando para cubrir todas las necesidades que teníamos. (...) Entonces mi tía, me crío a mí, a los hijos de ella, a mis hermanitos, a todos nos crío, entonces era ella la que mantenía pendiente de todos nosotros" (Informante 1).

Estas relaciones pueden incluso compensar las dificultades y las condiciones objetivas de la vida y el contexto, tal como lo afirman DíazLlañes (2001).

La vivencia es diferente cuando los referentes se circunscribieron a la vida interior de las familias, concebidas como núcleos limitados a los padres (o quién hacía sus veces) y los hijos, sin aparecer otras relaciones que hicieran aportes significativos.

"Si, como que solo se dejaba para la intimidad de la casa, cuando pasaba eso no nos gustaba compartir... y ahora que tú haces la pregunta no me acuerdo bien si mi abuela supo de esos episodios [se refiere al maltrato entre los padres], yo asimilo que sí, mi mamá le tuvo que haber contado en algún momento" (Informante 3). 
"En estemomentoconvivo con miabuela, con la que siempre he convivido durante tanto tiempo (...), desde mis cuatro años prácticamente, nada más con ella. (...) mi familia está compuesta por ... por mi abuela. Mi abuela... mi abuela, por mis tíos así, por ejemplo de... No [son hijos de ella], son hermanos de ella y... más bien con ella, prácticamente solo ella y yo" (Informante 4).

El ideal sobre el acompañamiento de los padres, minimiza la contribución de otras personas en torno al bienestar, de modo que, a pesar de reconocerse como valiosas sus contribuciones, en los y las jóvenes persiste la sensación de deuda. Como se expresó en el grupo de discusión de Moravia "un tío no es lo mismo que el papá de uno, ellos como por hacer el favor a uno y ya, pues ellos tienen obligaciones y no van a coger la obligación con uno, sino que es como por colaborarle mientras tanto".

La ambigüedad entre la ilusión de tener una familia nuclear de padre-madre-hijos y reconocer la existencia de otros que soportaron y apoyaron el proceso de bienestar, se repite una y otra vez en las narraciones; lo cual hace pensar en los efectos que tienen sobre esta valoración, su cercanía a diversos tipos de familias y filiaciones.

En la época actual, las figuras significativas se extienden a espacios de socialización cada vez más colectivos y amplios: la escuela, los grupos juveniles, los grupos de intereses, e incluso las relaciones amorosas aporta elementos desde los cuales se revisa y refuta la socialización al interior de la familia. Una institución educativa puede reconocerse como corresponsable de la influencia positiva "para que por ejemplo no abandonáramos el colegio" (Informante 1); $o$ la intervención de una exnovia frente a la adicción, puede llevar a la decisión de iniciar el proceso de rehabilitación (Informante 4).

Una definición posible de la referencialidad que se acoja a la experiencia expresada en estas trayectorias, debe considerar también la tensión entre las expectativas sobre la responsabilidad familiar, en tanto institución claramente delimitada e individualizada, y la oportunidad de tener soporte de lazos que se extienden e involucran relaciones más allá del círculo familiar; más aún, si las concepciones desde las cuales estos jóvenes definen que la familia, incluyen no sólo papá, mamá e hijos, sino que "también pueden ser las personas de las cuales tú estás rodeado" (Informante 1); de quienes esperan lazos de afecto, apoyo constante y reconocimiento.

Esta idea de familia atravesada por el afecto y el deseo, es una concepción históricamente reciente. Valdivia (2008), manifiesta que el cambio entre las nociones de familia, responde a diversos modelos conceptuales, en los cuales se da mayor prelación a una de las dimensiones: económicas, religiosas, socio-demográficas, legales o afectivas. Builes-Correa y BedoyaHernández (2008) concuerdan, al evidenciar que ha pasado de ser concebida como sistema público a ser vivida como una demanda afectiva, producto de la vida citadina, que ha convertido las relaciones familiares en un universo privado. Lo cual hace reconocer la pretensión de la vida contemporánea de proyectar en los padres la función socializadora y afectiva, dirigida a los hijos.

Tanto las expectativas de los y las jóvenes, como las demandas institucionales ejemplificadas en el Plan Estratégico de Juventud (2003-2013), hacen un reclamo por la ausencia de los padres. Este hecho encaja con que las expectativas frente a la familia en la actualidad, se centran en responsabilizarla del éxito o fracaso de los proyectos individuales de sus miembros, debido a lo cual las dificultades familiares son evaluadas de forma negativa o como fallas morales; el individuo, al ver su malestar o sentirse impedido frente a la autosuperación, tiene como alternativa señalar a los padres y a las maltrechas relaciones familiares como los causantes de su sufrimiento (Illouz, 2007).

Actualmente es muy difícil mantener al núcleo familiar diferenciado claramente de otras relaciones establecidas con el contexto inmediato. La linealidad que podría entenderse entre la socialización primaria y la secundaria descritas por Berger y Luckman (2012), parece romperse; otras relaciones empiezan a tener importancia para los sujetos antes de lo que se había teorizado. Lo familiar y lo extrafamiliar 
pueden solaparse e incluso invadirse, la familia está en déficit frente a la sobre exposición de estímulos propios de la socialización contemporánea (Gergen, 2010), existen nuevos valores y sentidos más amplios provenientes de otros medios de socialización, que inciden en los procesos de subjetivación de niños, niñas y adolescentes (Erazo-Caicedo \& DuitamaZamora, 2015).

\section{Referencialidad como cualidad de las relaciones}

La referencialidad se inscribe en doble vía y se da en forma simultánea. En el medio se disponen de condiciones socioeconómicas, familiares y comunitarias, las cuales proveen posibilidades de referenciación para los sujetos que nacen y crecen en éste; al mismo tiempo, el sujeto se adhiere a ciertos vínculos y cualidades en las personas con las que interactúa, que le permiten construir su idea de bienestar.

En los casos de los entrevistados, la constitución de la referencialidad asumió dos formas: a través de la relación con la madre o con ambos padres, o mediada por el reconocimiento y el apoyo recibido de varias personas vinculadas o no a la vida familiar. La ampliación de las relaciones logra que asuman diversas posiciones, contemplen variadas opciones, extiendan sus panoramas de actuación y construyan su autonomía. En contraposición al riesgo que implicó un vínculo familiar ideal que primó sobre otro tipo de relaciones posibles en el contexto, la multiplicidad de vínculos se estableció como oportunidad de bienestar en contextos en los que las condiciones fueron adversas. Esta observación coincide con López, Santos, Bravo y Valle (2013), quienes señalan los vínculos de confianza como potencial para compensar situaciones de separación y pérdida en menores en protección.

Los referentes vienen siendo personas significativas, en algunos casos familiares, pero no exclusivamente, quienes a través de sus capacidades, interés e insistencia en mantener la cercanía con los y las jóvenes, se presentan como garantes de la posibilidad de construir bienestar. Si bien, el reconocimiento del referente se hace a partir de diferenciar ciertas cualidades atribuidas a las personas, la constitución de la referencialidad aparece en las relaciones, que son descritas como oportunidades de reconocimiento $\mathrm{y}$ apoyo moral.

La multiplicidad relacional configura una red referencial, que cumple el papel social de dar soporte a la construcción de valoraciones positivas frente a la vida y las dificultades, sin negar la existencia de las influencias provenientes de la familia, del contexto o de la construcción individual de la experiencia. Es más, la red referencial hace parte de los anclajes de pertenencia que la familia puede establecer en un grupo social más amplio, y frente a condiciones de idealización que refuerzan las fallas de los padres, podría actuar en forma más propositiva, disponiendo de otras posibilidades de identificación para los y las jóvenes.

En tanto la referencialidad se basa en el reconocimiento y el apoyo, incluso la observación de las condiciones difíciles, podría ofrecer para los y las jóvenes la posibilidad de encontrar elementos favorables para su desarrollo personal. Lo que se requiere, en este sentido es que estén a la mano la provisión de relaciones suficientes, en las cuales no se corra el riesgo de que alguien pueda estar invisibilizado o aislado de los vínculos necesarios.

Poner en primer lugar el ideal de familia, el ideal de vida o el ideal de bienestar, ha hecho que en un contexto como el antioqueño, y en especial en la ciudad de Medellín, los valores centrados en la satisfacción del deseo del individuo se conviertan en parámetros normativos, desde los cuales las dificultades, la lentitud y los retos de la vida cotidiana son vividos como un déficit. Valoraciones totalitarias de este tipo, pueden conllevar una subvaloración de la experiencia real, desde la cual los y las jóvenes tienden a reconocerse en estados de malestar.

Con relación a la responsabilidad de los padres, es idealista pensar que en todos los casos un solo individuo, o a lo sumo dos, pueden llegar a reunir las cualidades requeridas para establecer relaciones de referencialidad que contribuyan al bienestar; más aun teniendo en cuenta las tensiones antes mencionadas. La posibilidad de diferentes relaciones, heterogéneas, con varios tipos de personas, en diversos escenarios y con 
distintos grados de intimidad, daría cuenta de mayores oportunidades para la construcción del bienestar. En este sentido, las relaciones se constituyen en opciones, y en tanto más amplitud tenga la red referencial, mayor dotación de herramientas a disposición de los y las jóvenes para relacionarse con el conflicto y la adversidad.

La sociedad tendría que garantizar que los niños, las niñas, los jóvenes y las jóvenes tuvieran a su disposición este tipo de despensa ${ }^{3}$ relacional, de modo que la responsabilidad frente a su bienestar no sea encargada de manera unilateral a los padres o al esfuerzo individual. En tanto se reconocen las limitaciones de este estudio, por el tiempo de realización y en el número de participantes, los resultados son un abrebocas frente a la aplicación del concepto de referencialidad en el análisis de otras trayectorias, en diversas situaciones de adversidad, oportunidad y resiliencia. El aprendizaje sobre las condiciones que influyen en la construcción subjetiva del bienestar y la forma como las redes de relaciones pueden aportar a ello, es un campo poco explorado; sin embargo, invita a extender sus implicaciones a la comprensión de las formas como se involucran familia y soporte social en los estudios que orientan políticas públicas de familia, infancia y adolescencia.

\section{Lista de referencias}

Alcaldía de Medellín (2003). Plan Estratégico Municipal de Juventud 2003-2013. Medellín: Alcaldía de Medellín.

Arias, A., Arias, M., Jaramillo, C., Restrepo, F. \& Ruiz, D. (2009). Relaciones sociales entre jóvenes universitarios: una mirada desde las lógicas subjetivas. Sabaneta: Autor.

Berger, P. \& Luckman, T. (2012). La construcción social de la Realidad. Buenos Aires: Amorrortu.

Bilbao, M. A., Techio, E. M. \& Páez, D. (2007). Bienestar subjetivo, cultura y valores

3 Despensa alude en sentido metafórico, a la provisión de relaciones consideradas como nutrientes para la vida emocional y el bienestar, constituyéndose en inventario a proveer, cuidar y conservar desde la vida social y comunitaria. personales: estado de la cuestión y síntesis meta-analítica de estudios. Revista de Psicología, 25 (2), pp. 233-276.

Builes-Correa, M. V. \& Bedoya-Hernández, M. (2008). La familia contemporánea: relatos de resiliencia y salud mental. Revista Colombiana de Psiquiatría, 37 (3), pp. 344-354.

Carballeira, M., González, J. A. \& Marrero, R. J. (2015). Diferencias transculturales en bienestar subjetivo: México y España. Anales de Psicología, 31 (1), pp. 199-206. Doi.org/10.6018/analesps.31.1.166931

Cardona-Rodríguez, F. J., Osorio-Tamayo, D. L. \& Moreno-Carmona, N. D. (2014). Construcción del bienestar juvenil en las actuales dinámicas de socialización. Revista Colombiana de Ciencias Sociales, 5 (1). Doi: http://dx.doi. org/10.21501/22161201.959

Carrero, V., Soriano, R. \& Trinidad, A. (2012). TeoríaFundamentada "Grounded Theory". La Construcción de la teoría a través del análisis interpretacional. Madrid: Centro de Investigaciones Sociológicas.

Díaz-Llañes, G. (2001). El Bienestar subjetivo. Actualidad y perspectivas. En Revista Cubana de Medicina General Integral, 17 (6), pp. 172-179.

Erazo-Caicedo, E. D. \& Duitama-Zamora, Z. (2015). Aproximación a la construcción de las concepciones de infancia y juventud y su impacto en los procesos de subjetivación infantil y juvenil contemporáneos. Revista Perspectivas Educativas, 4, pp. 81-102.

Recuperado de: 07/06/2016 http://revistas. ut.edu.co/index.php/perspectivasedu/ article/download/705/548

Flick, U. (2004). Introducción a la investigación cualitativa. Madrid: Morata.

Galeano, M. E. (2004). Diseños de proyectos en la investigación cualitativa. Medellín: Fondo Editorial Eafit.

Gergen, K. J. (2010). El Yo Saturado. Dilemas de la identidad en el mundo contemporáneo. Barcelona: Paidós Ibérica.

Gómez, V., Villegas, C., Barrera, F. \& Cruz, J. (2007). Factores predictivos del bienestar subjetivo en una muestra colombiana. 
Revista Latinoaméricana de Psicología, 39 (2), pp. 311-325.

Hillman, J. (1998). El Código del Alma. Barcelona: Martínez Roca.

Illouz, E. (2007). Identidades Congeladas. Buenos Aires: Kats Editores. Recuperado de: http://www.katzeditores.com/images/ fragmentos/illouz.pdf.

López, M., Santos, I., Bravo, A. \& Valle, J. F. (2013). El proceso de transición a la vida adulta de jóvenes acogidos en el sistema de protección infantil. Anales de Psicología, 29 (1), pp. 187-196. Doi.org/10.6018/ analesps.29.1.130542

López-Sánchez, F. (2015). Adolescencia. Necesidades y problemas. Implicaciones para la intervención. Adolescere, 3 (2), pp. 9-17. Recuperado de: http://www. adolescenciasema.org/wp-content/ uploads/2015/06/adolescere-2015vol3-n2_9-17_Adolescencia.pdf

Montoya-Zuluaga, D. M., Castaño-Hincapié, N. \& Moreno-Carmona, N. D. (2016, enero-junio). Enfrentando la ausencia de los padres: recursos psicosociales $\mathrm{y}$ construcción de bienestar. Revista Colombiana de Ciencias Sociales, 7 (1), pp. 181-200.

Páez-Martínez, R. M. (2016). ¿Des-víos de la familia en la formación de los hijos? Nuevos perfiles, funciones constantes. Revista Latinoamericana de Ciencias Sociales, Niñez y Juventud, 14 (1), pp. 261-272. Doi: 10.11600/1692715x.14117260215.

Quiceno, N., Cardona, J. \& Montoya, H. (2006). Moravia una historia de resiliencia. Medellín:Alcaldía de Medellín. Recuperado de: http://www.medellindigital.gov.co/ Mediateca/repositorio\%20de\%20recursos/ AlcadiaMedellin_Moravia_Resistencia. pdf

Strauss, A. \& Corbin, J. (2002). Bases de la investigación cualitativa. Técnicas $y$ procedimientos para desarrollar la teoría fundamentada. Medellín: Editorial Universidad de Antioquia.

Valdivia, C. (2008). La familia: concepto, cambios y nuevos modelos. La Revue $d u$ Redif, 1, pp. 15-22.
Vielma, J. \& Alonso, L. (2010). El estudio del Bienestar Psicológico Subjetivo. Una breve revisión teórica. Educere, 14 (49), pp. 265-275. 